\title{
Linx
}

Revue des linguistes de l'université Paris X Nanterre

$58 \mid 2008$

Aspects de comme

\section{Présentation : Aspects de comme}

\section{Marianne Desmets, Antoine Gautier et Thomas Verjans}

\section{OpenEdition}

\section{Journals}

Édition électronique

URL : http://journals.openedition.org/linx/321

DOI : 10.4000/linx.321

ISSN : 2118-9692

\section{Éditeur}

Presses universitaires de Paris Nanterre

\section{Édition imprimée}

Date de publication : 1 juin 2008

Pagination : 7-9

ISSN : 0246-8743

\section{Référence électronique}

Marianne Desmets, Antoine Gautier et Thomas Verjans, «Présentation : Aspects de comme », Linx [En ligne], 58 | 2008, mis en ligne le 15 février 2011, consulté le 22 septembre 2020. URL : http:// journals.openedition.org/linx/321 ; DOI : https://doi.org/10.4000/linx.321 


\title{
Présentation : Aspects de comme
}

\author{
Marianne Desmets - \\ Université Paris-Ouest-UMR 7110-LLF E UMR 7114-MODYCO \\ Antoine Gautier - Université Paris-Sorbonne-EA 4089-STIH \\ Thomas Verjans - Université Paris-Sorbonne-EA 4089-STIH
}

Ce numéro, intitulé «Aspects de comme», est issu de travaux menés dans le cadre du projet Gram- $M^{1}$. L'objectif initial de ce groupe de recherche fut double. Ce fut, d'une part, d'établir un état des connaissances sur comme afin d'obtenir une vue globale de ce terme si labile, en confrontant à la fois différentes études descriptives existantes, et en produisant un certain nombre d'études originales visant à éclairer des aspects moins bien repérés dans la littérature. D'autre part, l'originalité du projet fut de réunir des chercheurs provenant de plusieurs horizons théoriques; la confrontation critique des modèles convoqués (grammaire syntagmatique, approche constructionnelle, psychomécanique du langage) permettant de saisir en quoi leur exploitation permettait de rendre compte des multiples aspects du mot comme, de situer celui-ci dans l'ensemble plus vaste des systèmes auxquels il appartient, tant du point de vue interne que du point de vue externe.

Le présent recueil se veut ainsi complémentaire des études contemporaines ${ }^{2}$ qui visent à recenser les différents types d'emploi assumés par comme. Dans la perspective d'appréhender un large spectre du fonctionnement de ce terme, nous avons regroupé

\footnotetext{
${ }^{1}$ Groupe de travail réunissant jeunes chercheurs et chercheurs confirmés, créé par A. Gautier et Th. Verjans, affilié à l'équipe Sens, Texte, Informatique et Histoire (Paris-Sorbonne), et consacré à l'étude des mots grammaticaux.

2 Notamment Flaux \& Moline (2008).
} 
les travaux en deux ensembles, selon que la perspective des contributions est synchronique ou diachronique, chacun des deux ensembles étant ouvert par une synthèse générale constituant un état des lieux des principaux travaux menés sur la question. En outre, nous avons délibérément choisi des contributions d'arrière-plans théoriques variés, et qui portent un intérêt tout particulier à comme en regard des relations systématiques qu'il entretient non seulement entre ses différents emplois, dont l'emploi comparatif, que l'on peut à bon droit tenir pour prototypique, mais aussi avec d'autres marqueurs, conjonctions ou proformes.

Dans la perspective synchronique, Antoine Gautier propose un parcours de l'ensemble des travaux de façon à isoler les principaux problèmes suscités par comme, et tout particulièrement le problème de son appartenance catégorielle et le problème de la possibilité d'une description unifiée de ses valeurs d'emploi. La section consacrée aux contributions diachroniques se fonde principalement sur une périodisation des emplois de comme, et s'ouvre à une réflexion épistémologique sur les mérites et manques d'une telle démarche.

Dans la perspective synchronique, l'article d'Estelle Moline interroge d'abord l'emploi exclamatif de comme au prisme de la réflexion menée sur les proformes indéfinies. Partant de l'hypothèse que comme est, dans cet emploi, un adverbe de manière, l'article met en évidence un ensemble de propriétés spécifiques qui distinguent comme des autres proformes exclamatives. Marianne Desmets s'attache ensuite à la question de l'ellipse dans les constructions comparatives en comme. Optant pour une approche constructionnelle, elle isole ainsi les propriétés de ces séquences fragmentaires et montre le caractère «trans-constructionnel» attaché à ce phénomène. François Mouret, en collaboration avec Marianne Desmets, interroge la valeur coordonnante de comme dans les suites $S N$ comme $S N$ en position sujet et avec accord du verbe au pluriel. Ces auteurs montrent ainsi que, dans ces emplois, proches des comparatives d'analogie mais s'en distinguant pragmatiquement et prosodiquement, comme doit être recatégorisé en tant que conjonction de coordination. Les différentes analyses sont présentées dans le cadre d'une grammaire syntagmatique. Els Tobback et Bart Defrancq examinent, quant à eux, le cas de comme introduisant un attribut de l'objet. Choisissant de recourir à une approche constructionnelle, ils mettent en évidence les propriétés qui déterminent l'apparition du morphème dans ces configurations et qui en font une structure marquée, par opposition à la structure non marquée sans comme, des facteurs catégoriels et informationnels décidant de l'emploi de l'un ou de l'autre de ces deux «allostructions ». Enfin, Antoine Gautier et Mélanie Morinière, qui s’inscrivent également dans le cadre d'une approche de type constructionnelle, s'attachent à l'interprétation des segments comme $N$, en isolant ce qui relève de l'invariant sémantique et des propriétés micro-sémantiques de comme de ce qui tient aux facteurs contextuels mis en jeu, les plus importants étant en l'occurrence la structuration informationnelle de l'énoncé et la position du segment dans la phrase.

Les contributions diachroniques qui constituent la seconde partie de ce volume font d'abord écho aux précédentes. Ainsi, Mélanie Morinière, prolongeant l'article en collaboration avec A. Gautier, se concentre sur l'évolution des emplois du segment comme $N$, en montrant d'abord que comme peut, dès l'ancien français, assumer le rôle d'indicateur fonctionnel, mais que ce n'est que bien plus tardivement qu'il a pu 
être employé dans des structures à renversement de l'orientation informationnelle, proche en cela d'un circonstant de domaine. Enfin, elle parvient à dater du début du $\mathrm{XX}^{\mathrm{e}}$ siècle l'apparition de l'emploi non paradigmatique de comme. Th. Ponchon, dans le cadre de la psychomécanique du langage, s'intéresse à la valeur de com(e) en français médiéval lorsqu'il est suivi d'un adjectif. Mettant en lumière la double incidence à laquelle répond $\operatorname{com}(e)$ dans ce type d'emplois, il argumente ainsi en faveur d'une catégorisation en préposition, emploi spécifique au français médiéval et qui tend à disparaitre à l'aube du siècle renaissant. Enfin, Thomas Verjans aborde les dérivations à partir de com(e), et plus particulièrement le cas d'un adverbe relativement méconnu : cumfaitement. Celui-ci, apparu dès la Chanson de Roland, entre en concurrence avec com(e) et comment, particulièrement dans la série des emplois interrogatifs (interrogation directe et indirecte). Il se donne pour premier objectif de proposer une description syntactico-sémantique de cumfaitement, avant d'envisager plus précisément les rapports de concurrence qu'il entretient avec com(e) et comment. Par ailleurs, le fait que ce terme ne survive pas au-delà du XIII e siècle permet d'aborder la question du changement linguistique par le biais du phénomène des disparitions et, ce faisant, d'appréhender la façon dont une disparition se corrèle aux relations systématiques. 